\title{
An improved zero-dimensional model for simulation of TRIGA Mark II dynamic response
}

\author{
Carolina Introini ${ }^{\mathrm{a}}$, Antonio Cammi ${ }^{\mathrm{a},}{ }^{*}$, Stefano Lorenzi $^{\mathrm{a}}$, Giovanni Magrotti ${ }^{\mathrm{b}}$ \\ ${ }^{a}$ Department of Energy, Politecnico di Milano, Via La Masa 32, Milano, Italy \\ ${ }^{b}$ Laboratorio Energia Nucleare Applicata (LENA), University of Pavia, via Aselli 41, Pavia, Italy \\ *Corresponding author: antonio.cammi@polimi.it
}

\begin{abstract}
The primary aim of this work is to improve the analysis of the dynamic behaviour of the TRIGA Mark II reactor at the University of Pavia through a zero-dimensional approach. Besides the coupling between neutronics (point-reactor kinetics with six delayed neutron precursors group) and thermal-hydraulics (two-region model, with fuel and coolant) implemented in earlier works, the new model considers also the time behaviour of the mass flow rate due to natural circulation, of the neutron poisons and of the primary and secondary pool temperature. The system of coupled first-order differential equations is non-linear, as some state variables, such as the mass flow rate and the coolant temperature, multiply each other. The Simulink ${ }^{\mathrm{TM}}$ programming environment for dynamic analysis and control purposes is used to solve the system. A comparison with experimental data collected on-site for different reactor power transients and with measurements of the poison anti-reactivity during reactor shut-down and of the pool temperature allows the validation of the model. The model results and the experimental data reach a remarkable agreement. In addition, a linear stability analysis of the reactor is performed through the root locus and the stability map in terms of the thermal feedback coefficients. This analysis shows how the power level influences the dynamic of the system, and that, for certain values (always negative) of the fuel thermal feedback coefficient, positive values of the one for the moderator still ensures the system stability.
\end{abstract}

Keywords: TRIGA Reactor, Linear Stability Analysis, Reactor Modelling, Dynamic System Response

\section{Introduction}

In this work, the TRIGA Mark II research reactor of the University of Pavia is analysed through a zero-dimensional approach to highlight its dynamic behaviour, and to do a linear stability analysis, in the operational power range $(0-250 \mathrm{~kW})$.

The zero-dimensional model (also called lumped parameter approach) assumes spatial uniform quantities, which can be seen as average or weighted average values over the considered domain. This approach describes the behaviour of the distributed physical system in simpler terms, characterising the time transients in terms of spatial average 
quantities. The main drawback of such an approach is that it neglects local phenomena. On the other hand, dealing with a system of ODEs compared to one of PDEs is much simpler and faster, allowing for fast analysis of the reactor dynamic response. In addition, when adopting high-order modelling, spurious and numerical-induced behaviour and noise can alter how the model represents the real dynamics of the system, by producing non-natural effects. Zero-dimensional approaches do not have this issue (Christofides, 2001; Pitton and Rozza, 2017).

In an earlier work (Cammi et al., 2013) a point-reactor kinetics model with one energy group and six delayed neutron precursors, coupled with a two-region (fuel and coolant) thermal-hydraulics one, was developed. However, such a model considers neither the reactor pool and the secondary cooling system nor the effect of the neutron poisons on reactivity. This work fills the gap by improving the existing model. The rationale to include both the neutron poisons evolution and the secondary cooling system is the need to investigate not merely the short-term behaviour of the reactor during power transients but also the long-term one, meaning the one that occurs after the prompt effect (the fuel temperature feedback) following a reactivity insertion or withdrawal have exhausted their importance when compared to the delayed ones added in the present model. In terms of temporal scale, the long-term behaviour occurs after ten minutes following the reactivity change. Even during nominal operation, the concentration of the species with nonnegligible neutron absorption cross section varies, leading to a global variation of the amount of reactivity in the core. While this effect is not observed during the initial transient, it becomes significant as time goes on due to the neutron poisons pile-up and accumulation, of causing a variation in the reactor power. As for what regards the secondary cooling system, its role is to provide additional cooling to the primary pool, through forced convection, during the long-time reactor operation, to avert an excessive increase in the pool temperature.

Natural circulation is the only cooling mechanism of the system until the primary pool average temperature reaches a certain threshold. When this happens, the forced cooling system switches on, and forced convection becomes the primary cooling mechanism for the reactor pool. Despite this, natural convection remains the driving force for the flow through the core. In (Cammi et al., 2013), the free convection coolant mass flow rate through the core was estimated with a pressure drop balance between the overall distributed pressure losses and the driving pressure due to buoyancy. The present work improves this estimation by modelling the mass flow rate due to natural circulation through a momentum balance equation in the primary flow direction and by including in the model the time evolution of the average primary pool temperature.

A comparison between experimental data collected on the site about for different power transients and model results allows the validation of the latter. A digital acquisition system developed in-house by L.E.N.A. (Laboratorio Energia Nucleare Applicata) and connected with the Reactor Instrumentation and Control System (I\\&C) performed the data collection. Low power and high-power transients were studied and compared with the one found by the previous model, to highlight the differences in terms of the long-term behaviour of the reactor following a reactivity insertion. Linearization of the model around the steady equilibrium state of interest allows performing a linear stability analysis to study both the stability of the reactor at the different power transients considered, by the study of the 
characteristic roots of the linear system, and the stability in terms of the thermal feedback coefficients for reactivity, whose value depends from the state of the system itself.

The structure of the paper is as follows. Section 2 presents a detailed description of the reactor model, starting from the original one developed in (Cammi et al., 2013). The poison concentration model (Section 2.1) follows, along with the derivation of the equation for the mass flow rate (Section 2.2). Section 2.4 describes the so-called secondary system model, which includes the two pools and the heat exchangers connecting them and the environment (the city aqueduct). Having shown the model, Section 3 highlights the transient analysis, performed using the Simulink ${ }^{\mathrm{TM}}$ environment, and the validation through comparison with experimental data. Section 4 then shows the linear stability analysis.

\section{Reactor model}

The previous model (Cammi et al., 2013) adopts a point-reactor kinetics model with one energy group and six delayed neutron precursors groups to model the neutronic behaviour of the system. The main assumptions behind this approach are that the normalised neutron flux $\psi(\boldsymbol{x}, t)$ can be written by separating the space dependency from the time one and that the normalised precursors number $\eta(x, t)$ has a fixed spatial distribution. Indicating with $n_{0}$ the stationary neutron number and with $\eta_{i, 0}$ the stationary precursors number for the critical reactor, the two normalised variables are as follows:

$$
\psi(t)=\frac{n(t)}{n_{0}} \quad ; \quad \eta_{i}(t)=\frac{c_{i}(t)}{c_{i, 0}} \quad i=1, \ldots, 6
$$

The evolution of the normalised variables is:

$$
\left\{\begin{array}{c}
\frac{d \psi}{d t}=\frac{\rho(t)-\beta}{\Lambda} \psi(t)+\sum_{i=1}^{6} \frac{\beta_{i}}{\Lambda} \eta_{i}(t) \\
\frac{d \eta_{i}}{d t}=\lambda_{i}\left(\psi(t)-\eta_{i}(t)\right)
\end{array}\right.
$$

where $\rho(t)$ is the system reactivity, $\beta_{i}$ is the delayed neutron fraction for the $\mathrm{i}$-th precursor, and $\lambda_{i}$ is the decay constant of the $i$-th precursor. The input of the system is represented by the control rod height $h_{\text {rod }}$, which is controlled by the operator. The equation for reactivity reads as follows:

$$
\rho(t)=\alpha_{\text {rod }}\left(h_{\text {rod }}-h_{\text {rod }, 0}\right)+\alpha_{f}\left(T_{f}(t)-T_{f, 0}\right)
$$

where $\alpha_{\text {rod }}\left(h_{\text {rod }}-h_{\text {rod, } 0}\right)$ represents the reactivity inserted by the control rods and $\alpha_{f}$ is the fuel temperature feedback coefficient (Johnson et al., 2010) (the coolant temperature feedback coefficient was not considered in (Cammi et al., 2013) due to the low gradients expected for the coolant). This coefficient represents the coupling mechanism between 
neutronics and thermal-hydraulics, the latter modelled through a two-region (fuel and coolant) approach, based on the average temperature of fuel and coolant:

$$
\left\{\begin{array}{c}
\frac{d T_{f}}{d t}=\frac{P_{0} f}{\tau_{f} K} \psi(t)-\frac{T_{f}(t)-T_{c}(t)}{\tau_{f}} \\
\frac{d T_{c}}{d t}=\frac{P_{0}(1-f)}{\tau_{c} K} \psi(t)+\frac{T_{f}(t)-T_{c}(t)}{\tau_{c}}-\frac{2 c_{c} \Gamma(t)\left(T_{c}(t)-T_{c, i n}(t)\right)}{\tau_{c} K}
\end{array}\right.
$$

The term $K\left(T_{f}(t)-T_{c}(t)\right)$ represents the total heat exchanged between fuel and coolant. It is worth noting how, in (Cammi et al., 2013), both the core inlet temperature and the core mass flow rate were considered as parameters of the system, and not as state variables. A summary of the original model parameters can be found in Table 1 and 2 .

\begin{tabular}{ccc}
\hline Symbol & Quantity & Value \\
\hline$\beta$ & $\begin{array}{c}\text { Total delayed neutron } \\
\text { fraction }\end{array}$ & $730 \times 10^{-5}$ \\
$\Lambda$ & Invariant neutron mean life & $60 \mu s$ \\
\hline Group & $\beta_{i} / \beta$ & $\lambda_{i}, s^{-1}$ \\
\hline 1 & 0.042 & 3.01 \\
2 & 0.115 & 1.14 \\
3 & 0.395 & 0.301 \\
4 & 0.196 & 0.111 \\
6 & 0.219 & 0.0305 \\
\hline
\end{tabular}

Table 1: Numerical values used in the original point-kinetics and reactivity model.

\begin{tabular}{ccc}
\hline Symbol & Quantity & Value \\
\hline$c_{c}$ & Moderator specific heat capacity & $4185 \mathrm{Jkg}^{-1} \mathrm{~K}^{-1}$ \\
$f$ & Fraction of power deposited in the fuel & 1 \\
$\tau_{f}$ & Moderator time constant & $40.0719 \mathrm{~s}$ \\
$\tau_{c}$ & Fuel time constant & $0.3998 \mathrm{~s}$ \\
\hline
\end{tabular}

Table 2: Numerical values used in the original thermal-hydraulics model.

\subsection{Poison concentration model}

The operation of a nuclear reactor causes the production and accumulation of fission products from the burnup of the fuel. Some of these present a high neutron absorption crosssection and interact with the neutrons within the fuel, reducing their population and thus reducing the fission ratio. The overall effect of their accumulation is an unwanted negative feedback mechanism, which decreases the overall reactivity of the system and must be compensated by moving the control rods. Whereas this effect is negligible for short-term transients, it must be considered in long-term ones.

The two most relevant species of neutron poisons are xenon ${ }_{54}^{135} \mathrm{Xe}$ and samarium ${ }_{62}^{149} \mathrm{Sm}$. 
Not only these two isotopes present the highest neutron absorption cross-section, but they also have the highest fission yield; thus, their production and consumption during operation must be considered. The decay chains for the two considered poisons are as follows:

$$
\left\{\begin{array}{c}
{ }^{135} \mathrm{Te} \stackrel{\beta^{-19.2 s}}{\longrightarrow}{ }^{135} \mathrm{I} \stackrel{\beta^{-6.58 h}}{\longrightarrow}{ }^{135} \mathrm{Xe} \stackrel{\beta^{-9.17 h}}{\longrightarrow}{ }^{135} \mathrm{CS} \stackrel{\beta^{-2.3 e 6 y}}{\longrightarrow}{ }^{135} \mathrm{Ba} \\
{ }^{149} \mathrm{Nd} \stackrel{\beta^{-1.7 h}}{\longrightarrow}{ }^{149} \mathrm{Pm} \stackrel{\beta^{-53 h}}{\longrightarrow}{ }^{149} \mathrm{Sm}
\end{array}\right.
$$

Regarding ${ }^{135} \mathrm{Xe}$, it is produced by fission and from the decay of iodine ${ }^{135} I$, while it is consumed through neutron absorption and its own decay. Due to the small half-life of ${ }^{135} \mathrm{Te}$, iodine is considered being produced directly by fission, using the yield of tellurium as a source term. On the other hand, ${ }^{149} \mathrm{Sm}$ is a stable isotope, thus it is consumed only by neutron capture. With respect to the source term, the same assumption used for iodine has been made, due to the small half-life of ${ }^{149} \mathrm{Nd}$ with respect to that of ${ }^{149} \mathrm{Pm}$.

To study the dynamic behaviour of the system, it is necessary to use a coupled model for neutron kinetics and thermo-hydraulics to consider the variation of the neutron flux during the time evolution of the system. In this regard, and considering the initial value of the atom density of $\mathrm{U}_{235}$ (namely, $U_{5,0}$ ) the thermal neutron flux $\varphi(t)$ can be estimated using the following:

$$
\phi(t)=\frac{P(t)}{E_{\text {fiss }} \sigma_{\text {fiss }} U_{5}(t) V_{\text {fuel }}}=\frac{P_{0}}{E_{\text {fiss }} \sigma_{\text {fiss }} U_{5,0} V_{\text {fuel }}} \psi(t)=\psi(t) \phi_{0}
$$

where $\phi(t)$ is the neutron flux, $U_{5}(t)$ is the fuel concentration, $P_{0}$ is the steady-state reactor power, $\psi(t)$ is the normalised neutron density. The steady-state neutron flux $\phi_{0}$ can be estimated as follows:

$$
\phi_{0}=\frac{P_{0}}{E_{\text {fiss }} \sigma_{\text {fiss }} U_{5,0} V_{\text {fuel }}}
$$

Considering all source and consumption terms of the four isotopes mentioned above, Equation 9, and the balance equation for the fuel, the evolution of the concentration of the radioisotopes of interest can be written through the following system of equations:

$$
\left\{\begin{array}{c}
\frac{d I}{d t}=y_{I} \sigma_{f i s s} U_{5}(t) \psi(t) \phi_{0}-\lambda_{I} I(t) \\
\frac{d X e}{d t}=y_{X e} \sigma_{f i s s} U_{5}(t) \psi(t) \phi_{0}+\lambda_{I} I(t)-\left(\sigma_{a}^{X e} \psi(t) \phi_{0}+\lambda_{X e}\right) X e(t) \\
\frac{d P m}{d t}=y_{P m} \sigma_{f i s s} U_{5}(t) \psi(t) \phi_{0}-\lambda_{P m} P m(t) \\
\frac{d S m}{d t}=\lambda_{P m} P m(t)-\sigma_{a}^{S m} \psi(t) \phi_{0} \operatorname{Sm}(t) \\
\frac{d U_{5}}{d t}=-\sigma_{a}^{U} \psi(t) \phi_{0} U_{5}(t)
\end{array}\right.
$$


A summary of the model parameters used in the poison evolution model can be found in Table 3.

\begin{tabular}{ccc}
\hline Symbol & Quantity & Value \\
\hline$E_{f i s s}$ & Average energy per fission event & $3.2 \times 10^{-11} \mathrm{~kJ}$ \\
$V_{\text {fuel }}$ & Fuel volume & $3.58 \times 10^{-4} \mathrm{~m}^{3}$ \\
$y_{I}$ & Iodine fission yield & 0.0639 \\
$y_{P m}$ & Promethium fission yield & 0.0107 \\
$y_{X e}$ & Xenon fission yield & 0.00237 \\
$\lambda_{I}$ & Iodine decay constant & $2.87 \times 10^{-5} \mathrm{~s}^{-1}$ \\
$\lambda_{P m}$ & Promethium decay constant & $3.63 \times 10^{-6} \mathrm{~s}^{-1}$ \\
$\lambda_{X e}$ & Xenon decay constant & $2.09 \times 10^{-5} \mathrm{~s}^{-1}$ \\
$\sigma_{a}^{S m}$ & Neutron absorption cross section (samarium) & $4.1 \times 10^{-20} \mathrm{~cm}^{2}$ \\
$\sigma_{a}^{U}$ & Overall neutron absorption cross section (fuel) & $681 \times 10^{-24} \mathrm{~cm}^{2}$ \\
$\sigma_{a}^{X e}$ & Neutron absorption cross section (xenon) & $2.65 \times 10^{-18} \mathrm{~cm}^{2}$ \\
$\sigma_{f i s s}$ & Fuel fission cross section & $580 \times 10^{-24} \mathrm{~cm}^{2}$ \\
\hline
\end{tabular}

Figure 1 shows the time evolution of the poison concentrations. The Xenon decrease is due to the different weights of the terms in Equation 11 and 12. The initial increase is due to Xenon production due to fission. As its concentration increases, the consumption term due to neutron capture also increases, becoming dominant over the production ones. After a few hours, the rate of production of Xenon from Iodine and fission equals the rate of removal, and at this point, the Xenon concentration reaches a minimum. Then, it increases to a new equilibrium level. On the contrary, the concentration of Iodine increases because the production term is always dominant over the consumption one.
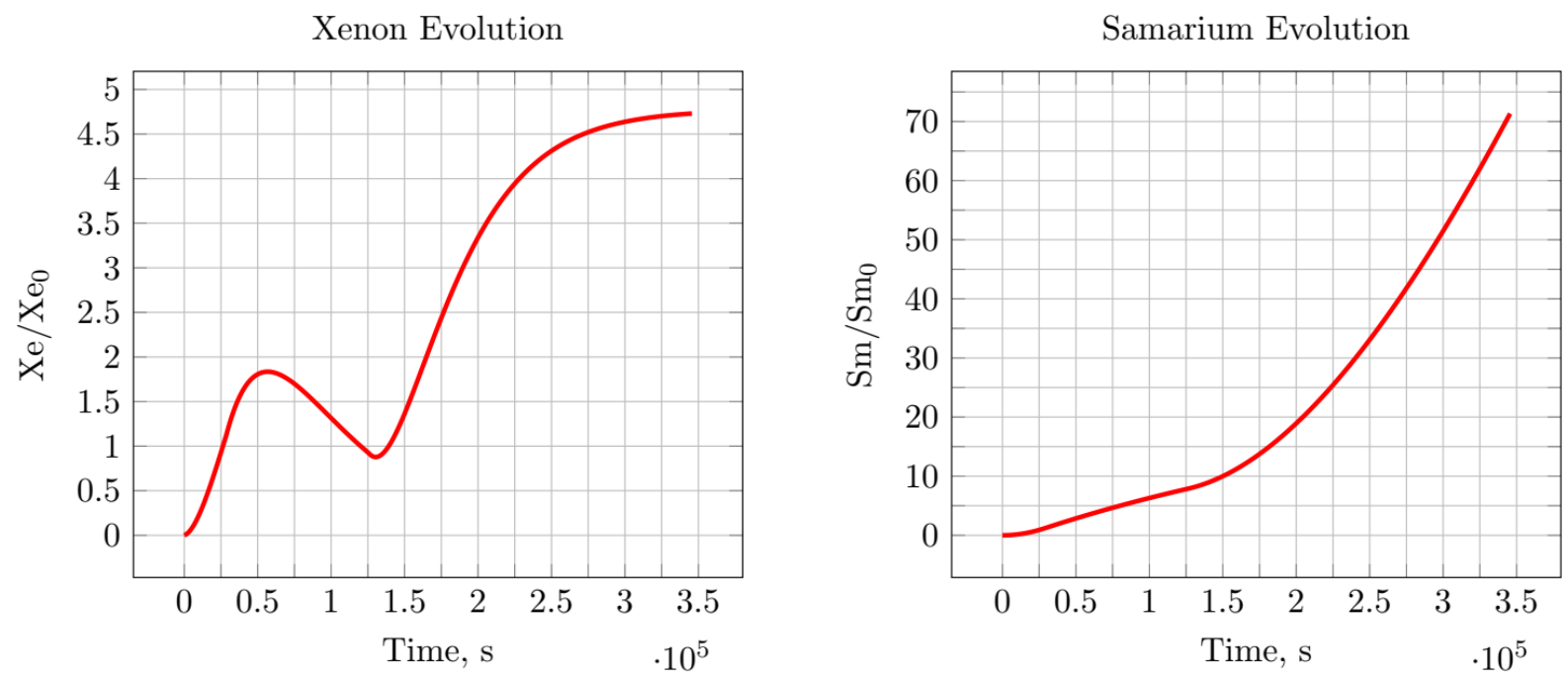

Figure 1: Time evolution of the normalised poison concentration for xenon (left) and samarium (right) for a long transient. As a reference, a power transient of $250 \mathrm{~kW}$ has been considered, starting from the cold reactor 
configuration (power around $1 \mathrm{~W}$ ) and withdrawing the control rods to obtain the desired power level.

As stated before, the overall effect of the accumulation of neutron poisons is a decrease of the overall reactivity of the system. In line with Equation 4, the negative feedback mechanism due to the time evolution of the neutron poisons concentration can be written by means of the feedback coefficient $\alpha_{X e}$ and $\alpha_{S m}$ (Zahedi and Ansarifar, 2018):

$$
\begin{gathered}
\rho_{X e}=-\frac{\sigma_{a}^{X e}\left(X e(t)-X e_{0}\right)}{\xi \sigma_{f i s s} U_{5}(t)}=\alpha_{X e}\left(X e(t)-X e_{0}\right) \\
\rho_{S m}=-\frac{\sigma_{a}^{S m}\left(S m(t)-S m_{0}\right)}{\xi \sigma_{f i s s} U_{5}(t)}=\alpha_{S m}\left(S m(t)-S m_{0}\right)
\end{gathered}
$$

Considering also the feedback effect due to variations of the coolant temperature (Zagar and Ravnik, 2005) and density, which were neglected in (Cammi et al., 2013) due to their secondary effect with respect to the fuel temperature feedback, the system reactivity for the TRIGA reactor can be written as:

$$
\begin{aligned}
\rho(t)=\alpha_{\text {rod }}( & \left.h_{\text {rod }}-h_{\text {rod, } 0}\right)+\alpha_{f}\left(T_{f}(t)-T_{f, 0}\right)+\alpha_{c}\left(T_{c}(t)-T_{c, 0}\right) \\
& +\alpha_{v}\left(\delta(t)-\delta_{0}\right)+\alpha_{X e}\left(X e(t)-X e_{0}\right)+\alpha_{S m}\left(S m(t)-S m_{0}\right)
\end{aligned}
$$

where $\alpha_{X e}=-\frac{\sigma_{a}^{X e}}{\xi \sigma_{f i s s} U_{5}(t)}$ and $\alpha_{S m}=-\frac{\sigma_{a}^{S m}}{\xi \sigma_{f i s s} U_{5}(t)}$ represent a feedback coefficient for the neutron poisons (respectively, Xenon and Samarium). Figure 2 represent the evolution of the anti-reactivity introduced by the poison accumulation. It is worth point out how this curve follows almost the same behaviour as the Xenon concentration. This means that the reactivity is mainly affected by Xenon, and the contribution given by Samarium may be neglected.

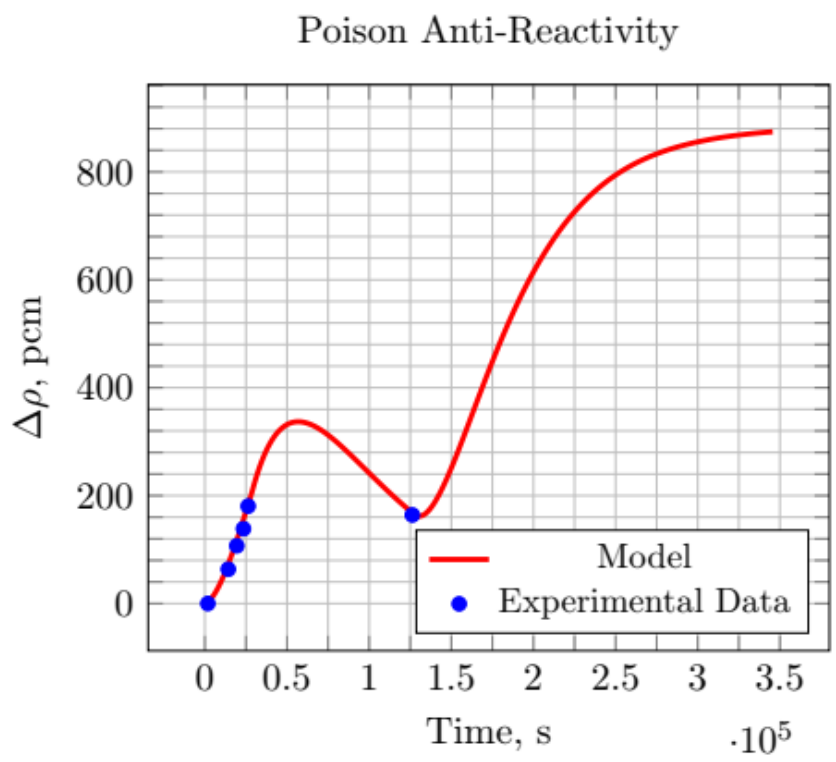

Figure 2: Time evolution of anti-reactivity, evaluated from the poison concentration. The blue points indicate 
experimental values, obtained from the control rods calibration curves. As a reference, a power transient of $250 \mathrm{~kW}$ has been considered, starting from the cold reactor configuration (power around $1 \mathrm{~W}$ ) and withdrawing the control rods to obtain the desired power level.

A summary of the model parameters used in the system reactivity equation can be found in Table 4.

\begin{tabular}{ccc}
\hline Symbol & Quantity & Value \\
\hline$\delta_{0}$ & Steady state coolant inlet density & $998.2284 \mathrm{~kg} \mathrm{~m}^{-3}$ \\
$S m_{0}$ & Steady state concentration of Samarium & $1.49 \times 10^{16} \mathrm{n} \mathrm{cm}^{-3}$ \\
$X e_{0}$ & Steady state concentration of Xenon & $15.26 \times 10^{14} \mathrm{n} \mathrm{cm}^{-3}$ \\
$\alpha_{c}$ & Coolant temperature feedback coefficient & $4 \times 10^{-5} \mathrm{~K}^{-1}$ \\
$\alpha_{f}$ & Fuel temperature feedback coefficient & $-8.5 \times 10^{-5} \mathrm{~K}^{-1}$ \\
$\alpha_{v}$ & Coolant void reactivity feedback coefficient & $-2.5 \times 10^{-6} \mathrm{~cm}^{-3}$ \\
$\xi$ & Average number of neutrons per fission event & 2.43 \\
\hline
\end{tabular}

Table 4: Numerical values used in the system reactivity equation.

\subsection{Natural circulation model}

With respect to (Cammi et al., 2013), in the following model the mass flow rate $\Gamma(t)$ is considered a state variable, and no longer a parameter of the system. Thus, an additional differential equation to describe the time evolution of this variable is needed. Starting from a 0 -D momentum balance on the coolant in the principal flow direction (namely, the vertical one), the following expression is derived:

$$
M_{c} \frac{d v}{d t}=-\alpha A_{f} \Gamma^{2}(t)+g L A_{f}\left(\delta_{\text {in }}-\delta_{\text {out }}\right)
$$

The right-hand side of Equation 19 represents the algebraic sum of all forces acting on a fluid moving with velocity $v$. The first term indicates the force due to friction along the core (total pressure losses), expressed in terms of the overall friction coefficient $\alpha$. The second term represents the effect of buoyancy, expressed in terms of the difference in density between the inlet and outlet sections of the core, under the Boussinesq approximation for buoyancy-driven flows. Localised pressure drops, such as the ones due to the supporting grids, are neglected.

Considering the definition of the thermal expansion coefficient $v=-\frac{\partial D}{D d T}$, for small temperature variations this expression can be rearranged to find a relation between density and temperature differences:

$$
\delta_{\text {in }}-\delta_{\text {out }}=\delta_{\text {in }} v\left(T_{\text {out }}-T_{\text {in }}\right)
$$

Recalling that the velocity $v$ can be linked to the mass flow rate through the relation $v=$ $\frac{\Gamma}{\rho A_{f}}$, it is possible to write a differential equation for the time behaviour of the mass flow rate $\Gamma(t)$ : 


$$
\frac{d \Gamma}{d t}=2 \delta_{\text {in }} v \boldsymbol{g} A_{f}\left(T_{c}(t)-T_{\text {in }}(t)\right)-\frac{\alpha A_{f}}{L_{\text {core }}} \Gamma^{2}(t)
$$

where $v$ is the magnitude of the fluid velocity, $v$ is the thermal expansion coefficient, taken as constant for the case of small temperature variations. A summary of the physical parameters used for the natural circulation model can be found in Table 5 .

\begin{tabular}{ccc}
\hline Symbol & Quantity & Value \\
\hline$A_{f}$ & Flow cross area & $0.0635 \mathrm{~m}^{2}$ \\
$\delta_{i n}$ & Steady state inlet density & $998.23 \mathrm{~kg} \mathrm{~m}^{-3}$ \\
$f_{D}$ & Darcy friction factor & 0.03 \\
$\boldsymbol{g}$ & Gravity acceleration & $9.81 \mathrm{~m} \mathrm{~s}^{-2}$ \\
$L_{\text {core }}$ & Total height of the core & $0.7224 \mathrm{~m}$ \\
$P_{w}$ & Wetted perimeter & $10.69 \mathrm{~m}$ \\
$v$ & Thermal expansion coefficient & $2.45 \times 10^{-4} \mathrm{~K}^{-1}$ \\
\hline
\end{tabular}

Table 5: Numerical values used in the natural circulation model

With respect to the friction coefficient $\alpha$, its derivation follows the assumption that the concentrated pressure losses can be neglected, because of the small value of flow velocity due to natural circulation. The frictional pressure losses within the core can be written, according to (Todreas and Kazimi, 1990), as:

$$
\Delta p_{f}=f_{D} \frac{L_{c o r e} P_{w}}{4 A_{f}}\left(\frac{\delta v_{r e f}^{2}}{2}\right)
$$

Expressing the velocity $v_{r e f}$ in terms of the mass flow rate through the core:

$$
\Delta p_{f}=f_{D} \frac{L_{c o r e} P_{w}}{4 A_{f}}\left(\frac{\Gamma^{2}}{2 \delta A_{f}^{2}}\right)
$$

The friction factor $f_{D}$ depends on flow geometry and flow regime. In this work, however, it has been taken as constant. In addition, since the expected density variation through the core is small, $\delta(t)$ has been taken as a constant equal to the inlet density, with good approximation. The frictional pressure losses along the core can then be written as:

$$
\Delta p_{f}=f_{D} \frac{L_{\text {core }} P_{w}}{8 \delta_{\text {in }} A_{f}^{3}} \Gamma^{2}
$$

Consequently, the coefficient $\alpha$ is defined as follows, and can be calculated using the parameters defined in Table 5: 


$$
\alpha=f_{D} \frac{L_{\text {core }} P_{w}}{8 \delta_{i n} A_{f}^{3}}
$$

\subsection{Heat transfer coefficient}

The heat transfer between fuel and coolant is modelled through an overall heat transfer coefficient $K$, which considers the whole heat exchange. This quantity can be evaluated by computing the temperature difference between fuel and coolant considering the overall thermal resistance between the two. This resistance is composed by several terms, namely, the resistance of the fuel itself, of the gap between fuel and cladding, of the cladding, and the one representing the convective heat transfer between the cladding outer surface and the coolant (Todreas and Kazimi, 1990):

$$
K=\frac{n_{f e} V_{f}}{\pi r_{f o}^{2}\left[\frac{1}{8 \pi k_{f}}+\frac{1}{2 \pi r_{g} h_{g}}+\frac{\ln \left(\frac{r_{c o}}{r_{c i}}\right)}{2 \pi k_{c}}+\frac{1}{2 \pi r_{c o} h_{c}}\right]}
$$

\begin{tabular}{ccc}
\hline Symbol & Quantity & Value \\
\hline$h_{g}$ & Gap heat transfer coefficient & $2550 \mathrm{Wm}^{-2} \mathrm{~K}^{-1}$ \\
$k_{c}$ & Cladding thermal conductivity & $228 \mathrm{Wm}^{-1} \mathrm{~K}^{-1}$ \\
$k_{f}$ & Fuel thermal conductivity & $17.58 \mathrm{Wm}^{-1} \mathrm{~K}^{-1}$ \\
$n_{f e}$ & Number of fuel elements & 83 \\
$r_{c i}$ & Cladding inner radius & $0.01794 \mathrm{~m}$ \\
$r_{c o}$ & Cladding outer radius & $0.0187 \mathrm{~m}$ \\
$r_{f o}$ & Fuel outer radius & $0.01791 \mathrm{~m}$ \\
$r_{g}$ & Gap radius & $0.0179 \mathrm{~m}$ \\
$P r$ & Coolant Prandtl number & 4.076 \\
$D_{e q}$ & Equivalent hydraulic diameter & $0.0238 \mathrm{~m}$ \\
$\mu$ & Coolant dynamic viscosity & $1 \times 10^{-3} \mathrm{~Pa} \mathrm{~s}$ \\
\hline
\end{tabular}

Table 6: Numerical values used for the evaluation of the heat transfer coefficient $\mathrm{K}$ 


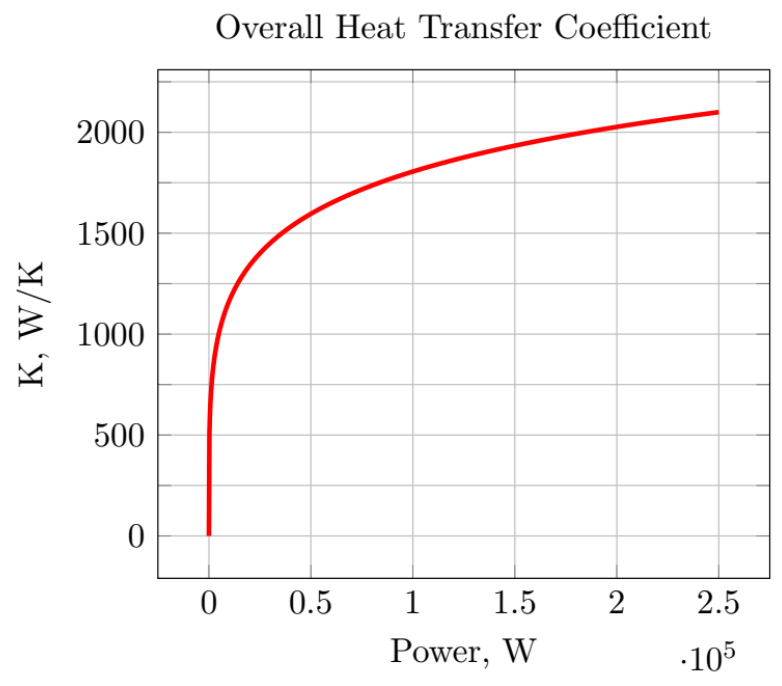

Figure 3: Overall heat transfer coefficient as function of the steady-state reactor power

The overall heat transfer coefficient shows a relevant dependence on the reactor power, especially at a low-power level, as seen in Fig. 3. This behaviour can be explained by the convective heat transfer resistance, which depends on $h_{c}$, the convective heat transfer coefficient This term further depends on the coolant flow condition and the core geometry. In the previous work, the Dittus-Boelter correlation (Dittus and Boelter, 1930), valid for turbulent flow in narrow channels, was used. However, the estimated flow regime within the reactor core lies outside the range of validity of this correlation, thus making its use not reliable. Natural circulation may occur at different flow regimes, and, the behaviour within the so-called transition region is not well known. In this work, to describe this region, the following correlation proposed by Ruiz (Ruiz et al., 2015) has been chosen:

$$
\left\{\begin{array}{c}
\lambda=\frac{64^{\Psi_{\lambda}} 0.316^{1-\Psi_{\lambda}}}{R e^{\Psi_{\lambda}+0.25\left(1-\Psi_{\lambda}\right)}} \\
\Psi_{\lambda}=\left[1+e^{\frac{R e-2530}{120}}\right]^{-1}
\end{array}\right.
$$

where $\lambda$ represent a friction coefficient, and $\Psi_{\lambda}$ is a factor to model the transition between laminar and turbulent flow without discontinuities. A similar strategy is adopted to evaluate the convective heat transfer coefficient. Two zones, laminar $(\operatorname{Re}<2500)$ and turbulent $(\operatorname{Re}>2500)$ were identified, and the developed correlation is (Pini, 2017):

$$
\left\{\begin{array}{c}
N u=N u_{L}{ }^{\Psi}\left(0.023 R e^{0.8} \operatorname{Pr}^{0.4}\right)^{1-\Psi} \\
R e=\frac{\Gamma(t) D_{e q}}{\mu A_{f}}
\end{array}\right.
$$

where $N u_{L}=4.36$ is the laminar Nusselt number, $0.023 \operatorname{Re}^{0.8} \mathrm{Pr}^{0.4}$ is the turbulent Nusselt number computed using the Dittus-Boelter correlation, $R e$ is the Reynolds number, which, being dependent on the coolant mass flow rate, is a function of time. Verification of this correlation can be found in (Pini, 2017). Table 6 lists the parameters used in the evaluation of 
the heat transfer coefficient.

\subsection{Pool and heat exchangers model}

The TRIGA Mark II heat removal system scheme is depicted in Figure 4. The primary pool surrounds, and it is directly heated by the core. Water from this pool is pumped to the first heat exchanger where it is cooled by the water coming from the secondary pool. This pool sends a mass of water also to the second heat exchanger, which sees on the other side water coming from the city aqueduct and dumped in the sewer. This double cooling system serves as a buffer in case of accidental changes of the aqueduct water temperature.

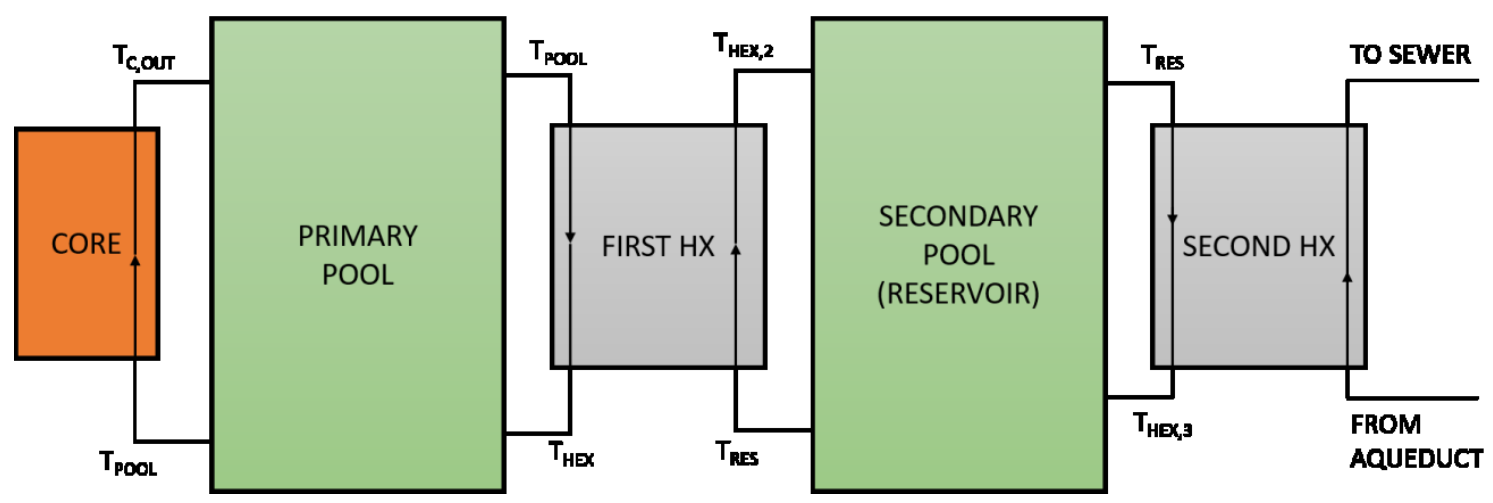

Figure 4: Pool and heat exchangers scheme for the TRIGA reactor.

\subsubsection{Primary pool model}

The primary pool surrounds the core, providing both a radiation shield and a water reservoir sufficiently large to minimise the temperature excursion of the coolant during transients. Water is taken from the top of the pool and sent to the primary heat exchanger. Then, the cooled-down water is re-injected just above the top of the core where it mixes with the flow of hot water that comes out the core itself due to natural circulation.

In the following, the pool is represented by its average temperature $T_{P}(t)$ and modelled through a simple energy balance:

$$
m_{p} c_{p} \frac{d T_{p}}{d t}=\Gamma(t) c_{p}\left(T_{c, \text { out }}(t)-T_{p}(t)\right)-\dot{m}_{1} c_{p}\left(T_{p}(t)-T_{h}(t)\right)
$$

By simplifying and rearranging the terms:

$$
\frac{d T_{p}}{d t}=\frac{\Gamma(t)}{m_{p}} T_{c, \text { out }}(t)+\frac{\dot{m}_{1}}{m_{p}} T_{h}(t)-\frac{\Gamma(t)+\dot{m}_{1}}{m_{p}} T_{p}(t)
$$

where $\Gamma(t)$ is the natural circulation flow rate within the core, $T_{c, \text { out }}(t)$ is the coolant temperature at the outlet section of the core, $T_{h}(t)$ is the temperature of the primary water out of the heat exchanger. Validation of the primary pool model can be seen in Figure 5. These data were obtained during a power transient at $200 \mathrm{~kW}$ (i.e., starting from the reactor operating at 
$200 \mathrm{~kW}$ and withdrawing the control rod to obtain a reactivity insertion of $30 \mathrm{pcm}$ ). The primary pool temperature was measured at the first heat exchanger inlet.

Average Primary Pool Temperature

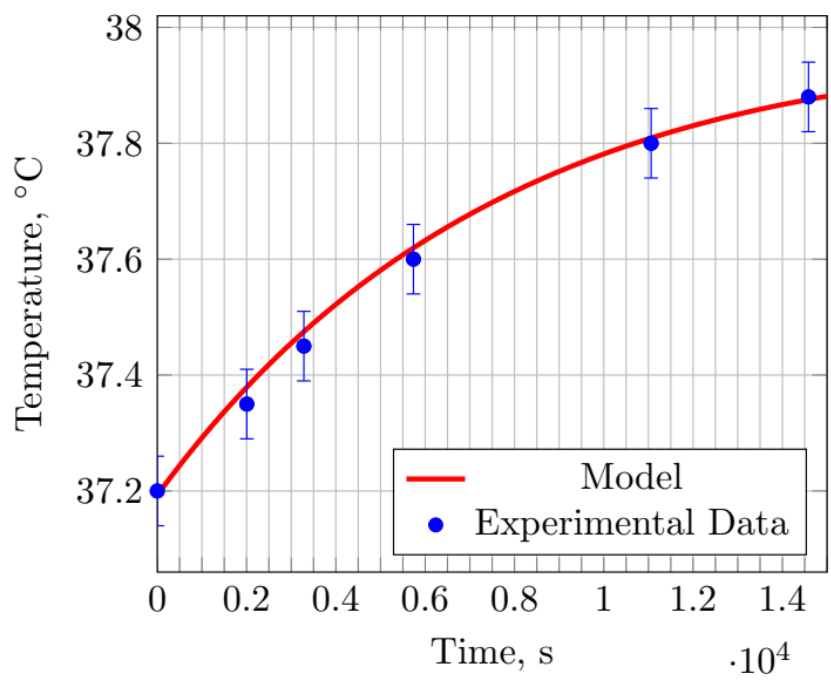

Figure 5: Validation of the primary pool model through comparison of experimental data and pool temperature evolution for a $200 \mathrm{~kW}$ power transient following reactivity insertion of $30 \mathrm{pcm}$.

\subsubsection{Secondary pool model}

The secondary pool provides the coupling between the primary and secondary heat exchanger and serves as a buffer during transients. From here, a mass flow rate is sent to the first heat exchanger where it cools down the primary water. Another mass flow rate is sent to the second heat exchanger. For simplicity, the two mass flow rates are modelled as constant and equal. Considering the average temperature of this reservoir $T_{r}(t)$ and its energy balance, the following equation is derived:

$$
\frac{d T_{r}}{d t}=\frac{\dot{m}_{2}}{m_{r}}\left(T_{h, 2}(t)-T_{r}(t)\right)-\frac{\dot{m}_{2}}{m_{r}}\left(T_{r}(t)-T_{h, 3}(t)\right)
$$

where $T_{h, 2}(t)$ is the temperature of the secondary water at the outlet of the first heat exchanger, $T_{h, 3}(t)$ is the temperature of the secondary water at the outlet of the second heat exchanger and $\dot{m}_{2}$ is the secondary water mass flow rate.

\subsubsection{Heat exchangers model}

The two heat exchangers are both shell-and-tube type, operating in counter-current mode. To model them, some preliminary assumptions are introduced:

- the heat exchangers are perfectly adiabatic, and no heat is dissipated to the environment;

- the behaviour of the heat exchangers is modelled using algebraic equations, and the variables of interest instantly reach steady-state conditions;

- the inlet temperature of the fluid with a greater mass flow rate is equal to the outlet temperature of the fluid with a smaller mass flow rate. This assumption, needed to close the 
system of equations, is justified by the high thermal capacity of water.

Through a straight-forward energy balance between hot and cold fluid, the equations for the two heat exchangers read:

$$
\left\{\begin{array}{c}
\dot{m}_{1} c_{p}\left(T_{p}(t)-T_{h}(t)\right)=\dot{m}_{2} c_{p}\left(T_{h, 2}(t)-T_{r}(t)\right) \\
\dot{m}_{2} c_{p}\left(T_{r}(t)-T_{h, 3}(t)\right)=\dot{m}_{3} c_{p}\left(T_{h, 4}(t)-T_{a q}(t)\right)
\end{array}\right.
$$

where $T_{h, 4}(t)$ is the temperature of the aqueduct water respectively at the outlet of the second heat exchanger. By introducing the assumptions made above, since $\dot{m}_{1}<\dot{m}_{2}$ and $\dot{m}_{2}$ $>\dot{m}_{3}$, Equations 34 and 35 become:

$$
\left\{\begin{array}{l}
\dot{m}_{1}\left(T_{p}(t)-T_{r}(t)\right)=\dot{m}_{2}\left(T_{h, 2}(t)-T_{r}(t)\right) \\
\dot{m}_{2}\left(T_{r}(t)-T_{h, 3}(t)\right)=\dot{m}_{3}\left(T_{r}(t)-T_{a q}(t)\right)
\end{array}\right.
$$

with $T_{a q}(t)$ taken as a constant input for the system. Now the mass flow rates in the heat exchangers are not constants, and their magnitude is controlled depending on the temperature of the water flowing in the primary heat exchanger (Boarin et al., 2016). However, to simplify the model, the values of the flow rates are kept constant in this work.

The model parameters used for the pool and heat exchanger model can be found in Table 7 .

\begin{tabular}{ccc}
\hline Symbol & Quantity & Value \\
\hline$m_{p}$ & Mass of water in the primary pool & $18963 \mathrm{~kg}$ \\
$m_{r}$ & Mass of water in the secondary pool & $800 \mathrm{~kg}$ \\
$\dot{m}_{1}$ & Primary flow rate in the primary HE & $7 \mathrm{kgs}^{-1}$ \\
$\dot{m}_{3}$ & Primary flow rate in the secondary HE & $7.6 \mathrm{kgs}^{-1}$ \\
$T_{a q}$ & Temperature of the aqueduct water & $15^{\circ} \mathrm{C}$ \\
\hline
\end{tabular}

\section{Transient analysis}

The dynamic behaviour of the reactor can now be described by the developed model: 


$$
\left\{\begin{array}{c}
\frac{d \psi}{d t}=\frac{\rho(t)-\beta}{\Lambda} \psi(t)+\sum_{i=1}^{6} \frac{\beta_{i}}{\Lambda} \eta_{i}(t) \\
\frac{d \eta_{i}}{d t}=\lambda_{i}\left(\psi(t)-\eta_{i}(t)\right) \\
\frac{d T_{f}}{d t}=\frac{P_{0} f}{\tau_{f} K} \psi(t)-\frac{T_{f}(t)-T_{c}(t)}{\tau_{f}} \\
\frac{d T_{c}}{d t}=\frac{P_{0}(1-f)}{\tau_{c} K} \psi(t)+\frac{T_{f}(t)-T_{c}(t)}{\tau_{c}}-\frac{2 c_{c} \Gamma(t)\left(T_{c}(t)-T_{c, i n}(t)\right)}{\tau_{c} K} \\
\frac{d \Gamma}{d t}=2 \delta_{\text {in }} v g A_{f}\left(T_{c}(t)-T_{\text {in }}(t)\right)-\frac{\alpha A_{f}}{L_{c o r e}} \Gamma^{2}(t) \\
\frac{d I}{d t}=y_{I} \sigma_{f i s s} U_{5}(t) \psi(t) \phi_{0}-\lambda_{I} I(t) \\
\frac{d X e}{d t}=y_{X e} \sigma_{f i s s} U_{5}(t) \psi(t) \phi_{0}+\lambda_{I} I(t)-\left(\sigma_{a}^{X e} \psi(t) \phi_{0}+\lambda_{X e}\right) X e(t) \\
\frac{d P m}{d t}=y_{P m} \sigma_{f i s s} U_{5}(t) \psi(t) \phi_{0}-\lambda_{P m} P m(t) \\
\frac{d S m}{d t}=\lambda_{P m} P m_{1}(t)-\sigma_{a}^{S m} \psi(t) \phi_{0} S m(t) \\
\frac{d U_{5}}{d t}=-\sigma_{a}^{U} \psi(t) \phi_{0} U_{5}(t) \\
\frac{d T_{p}}{d t}=\frac{\Gamma(t)}{m_{p}} T_{c, o u t}(t)+\frac{\dot{m}_{1}}{m_{p}} T_{h}(t)-\frac{\Gamma(t)+\dot{m}_{1}}{m_{p}} T_{p}(t) \\
\frac{\dot{m} T_{2}}{m_{r}}\left(T_{h, 2}(t)-T_{r}(t)\right)-\frac{\dot{m}_{2}}{m_{r}}\left(T_{r}(t)-T_{h, 3}(t)\right)
\end{array}\right.
$$

The above equation system is then rewritten in the following compact form:

$$
\left\{\begin{array}{l}
\dot{\boldsymbol{x}}=f(\boldsymbol{x}, \boldsymbol{u}) \\
\boldsymbol{y}=g(\boldsymbol{x}, \boldsymbol{u})
\end{array}\right.
$$

where $\boldsymbol{x}$ represents the state of the system, $\boldsymbol{u}$ the system input and $\boldsymbol{y}$ the system output:

$$
\left\{\begin{array}{c}
\boldsymbol{x}=\left[\begin{array}{llllllllllll}
\psi & \eta_{i} & T_{f} & T_{c} & \Gamma & I & X e & P m & S m & U_{5} & T_{p} & T_{r}
\end{array}\right] \\
\boldsymbol{y}=\left[\begin{array}{lllllllll}
\psi & \eta_{i} & T_{f} & T_{c} & T_{c, \text { out }} & \Gamma & \text { Xe } & \text { Sm } & T_{p}
\end{array}\right]
\end{array}\right.
$$

To study the dynamic behaviour of the reactor, the nonlinear system (39) of coupled ODEs has been solved using the Simulink ${ }^{\mathrm{TM}}$ environment (The MathWorks, Inc., 2008) (Figure 6).

The numerical solver chosen for the simulation is ode15s (Shampine, 1994), recommended for stiff problems. Validation of the model is performed through the comparison with experimental data of the reactor power during reactivity insertion transients collected in situ, for four different power transients at $50 \mathrm{~kW}, 100 \mathrm{~kW}, 150 \mathrm{~kW}$ and $200 \mathrm{~kW}$ as in (Cammi et 
al., 2013) (Figure 7). The experimental reactor power is computed from the neutron flux measured in the core during operation by means of the neutron activation technique (Borio di Tigliole et al., 2014). All transients start from the reactor at stationary conditions at the desired power level, following start-up. The control rods are then withdrawn to insert a fixed value of reactivity in the system (decided a priori). A new steady-state, at a slightly higher power level, is then reached. It can be clearly seen how the model is able to reproduce the dynamic behaviour of the system, with an overall maximum error less than 5\% (considering also the uncertainties associated with the experimental data and with the transient initial conditions).

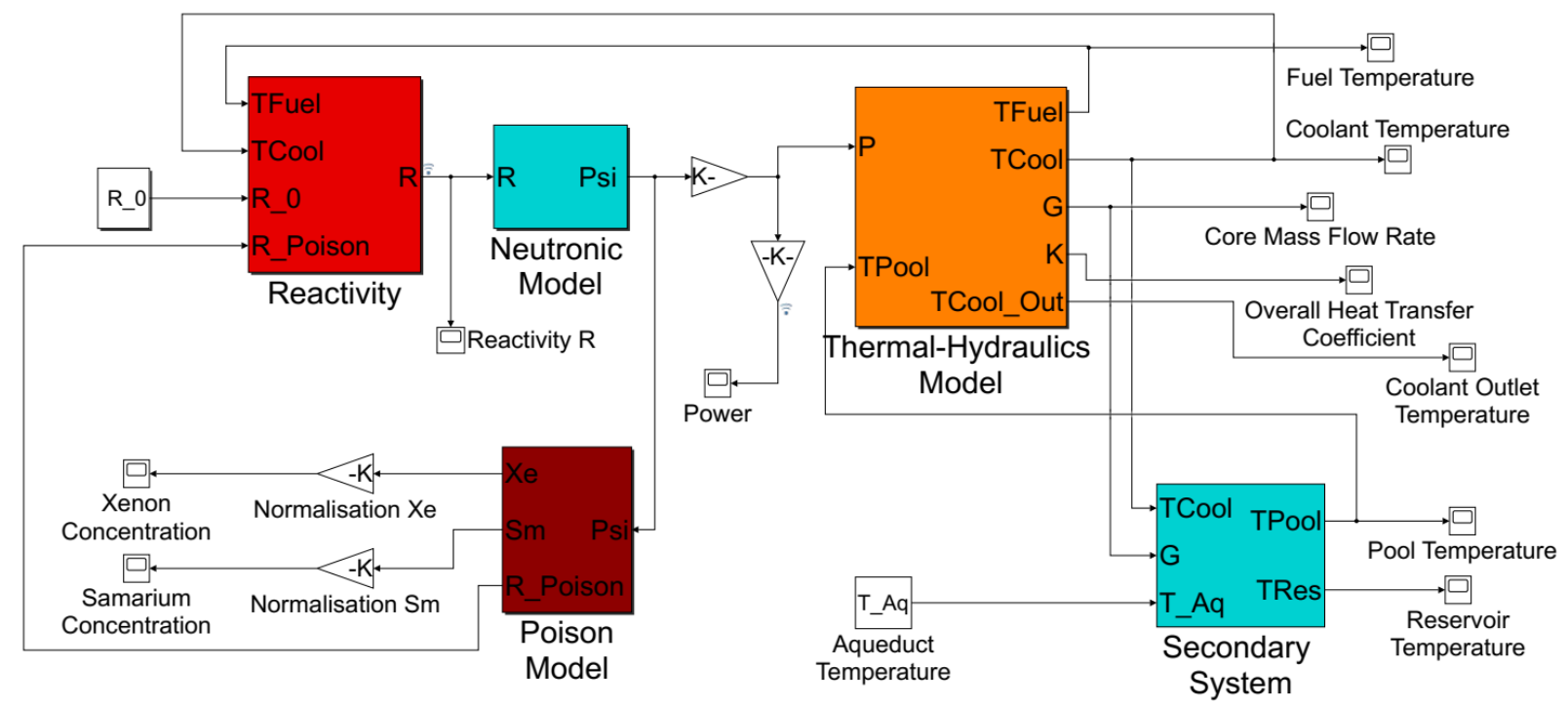

Figure 6: Simulink representation of the reactor model. The system components are represented through interconnected block diagrams, allowing an immediate insight of how the several components interact with each other and the dependencies among the different physics. The characteristic closed loop configuration can also be seen.

It is worth mentioning that the model predicts in all cases the position of the peaks later than the experimental data. These peaks represent the prompt effects given by the control rod withdrawal and the fuel feedback reactivity coefficient. The fact that all the positions of the peaks are predicted later than the experimental data might indicate that the prompt effects are faster than that predicted by the model. Evidently, there still are some reactivity prompt effects (both negative and positive) that the model does not consider.

Another source of error could be the fuel feedback coefficient, which in the following has been taken as a constant. This coefficient is a function of the reactor power. For the TRIGA reactor, due to the peculiar composition of the nuclear fuel (uranium in a matrix of moderating $\mathrm{ZrH}$ ), its determination is not straightforward, and it is affected by severe uncertainties (for example, linked also to the experimental procedure itself, which is not equivocally defined). For these reasons, in the following work, this coefficient has been taken as a constant. The same holds true also for the moderator coefficient, which may explain the slight discrepancies between experimental data and the end state for the transients.

Figure 8 shows the time behaviour of the system reactivity, both total and of the single contributions. Figure $8 \mathrm{a}$ describes the long-term transient (for sake of brevity, only the $200 \mathrm{~kW}$ one has been considered). General considerations are that, for the depicted behaviour, the most 
relevant feedback for the long term is the fuel temperature negative feedback, which, after a very sharp decrease due to the sudden reactivity insertion, continues to decrease thus causing a decrease in the reactor power. To counter-balance this effect and to maintain the system at the newly reached steady-state, the control rods need to be withdrawn to insert positive reactivity in the system. Both the coolant temperature and the poison absorption are secondary effects up to the considered time, however, the trend of the latter indicates that the poison effects will eventually become more relevant than the fuel one, to the point that the control rod movement will not be able to compensate it anymore.

(a) Reactor Power $(50 \mathrm{~kW})$

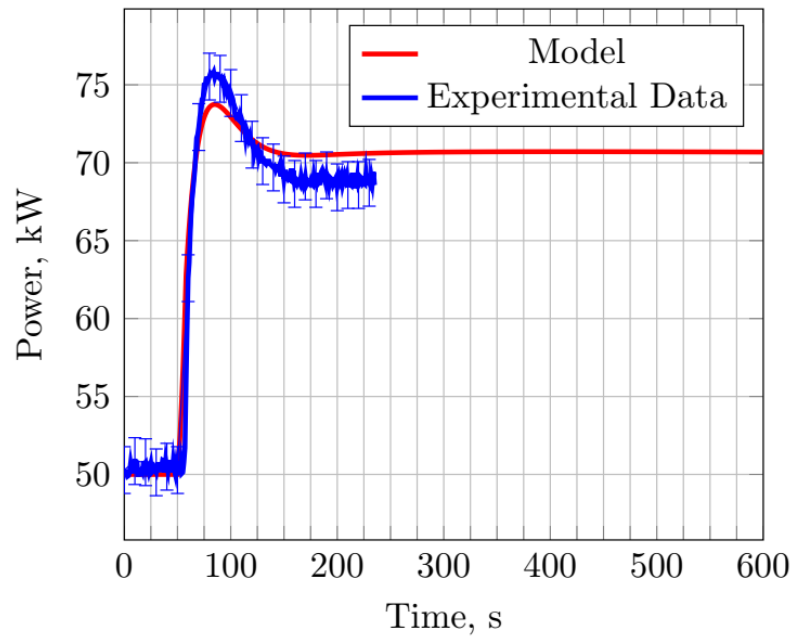

(c) Reactor Power $(150 \mathrm{~kW})$

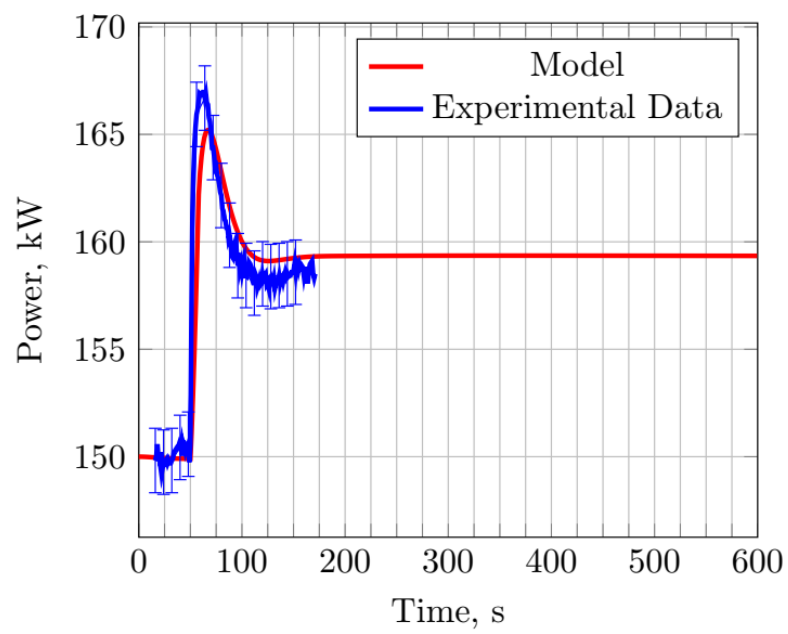

(b) Reactor Power $(100 \mathrm{~kW})$

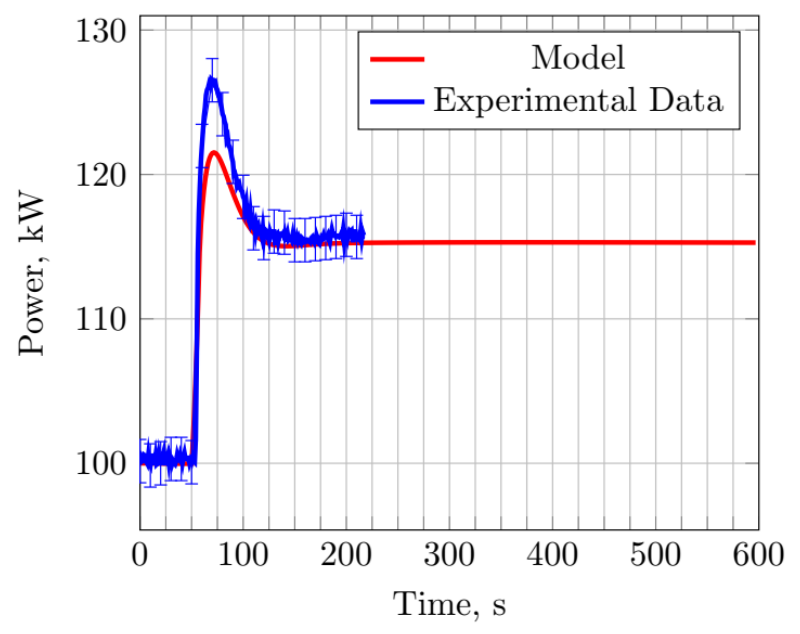

(d) Reactor Power $(200 \mathrm{~kW})$

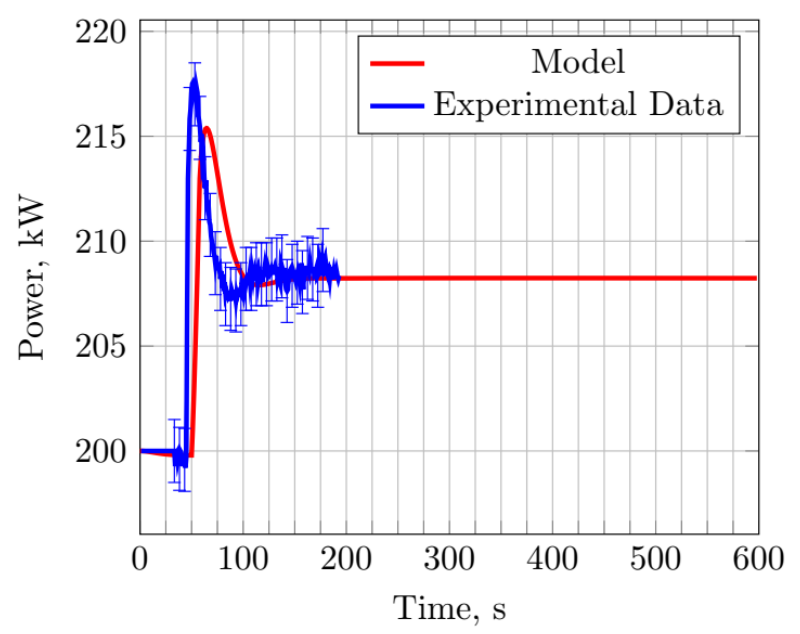

Figure 7: Model validation with respect to four different power transients following a reactivity insertion (control rod withdrawal). Clockwise from the top left corner: model response for reactivity insertion of $89 \mathrm{pcm}$ at $50 \mathrm{~kW}$ (a), reactivity insertion of $60 \mathrm{pcm}$ at $100 \mathrm{~kW}$ (b), $35 \mathrm{pcm}$ at $150 \mathrm{~kW}$ (c), $30 \mathrm{pcm}$ at $200 \mathrm{~kW}$ (d). Data were collected through a digital acquisition system connected with the reactor instrumentation and control system (I\\&C) and developed in-house. The total measurement uncertainty on the reactor power is around $1.5 \%$.

Figure $8 \mathrm{~b}$ highlights the short-term behaviour. Following reactivity insertion, the only visible prompt effect is that of the fuel temperature feedback, however it is slightly delayed with respect to reactivity insertion. This explains the presence of the power peak in the short- 
term (Figure 7), as the power increase is due to the positive insertion which, at the beginning, is not yet compensated by the fuel temperature feedback. Eventually, the response to the power increase is a negative contribution to the overall reactivity, thus decreasing the power level until the overall reactivity reaches zero and a new steady-state is reached.
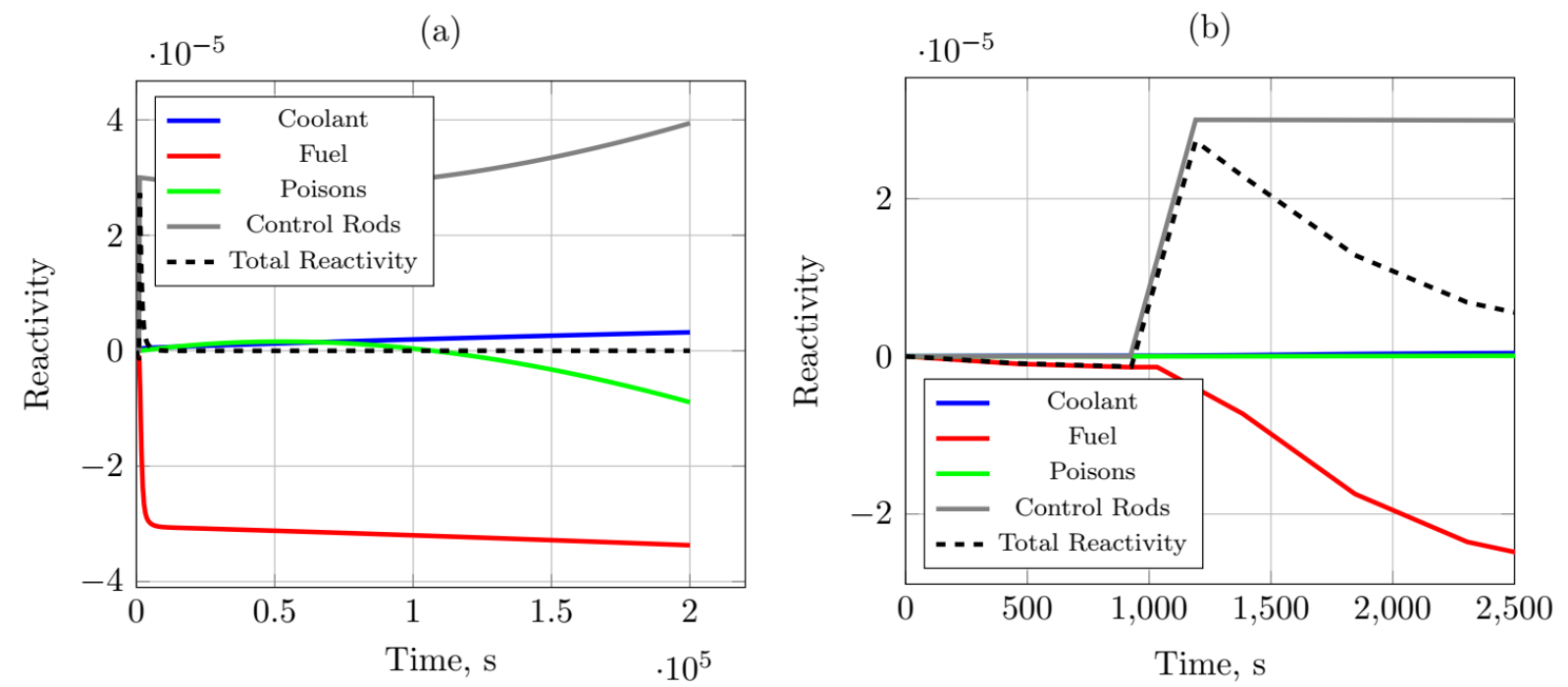

Figure 8: (a) Reactivity behaviour (total and components) for the long-term transient (as a reference, the $200 \mathrm{~kW}$ transient has been considered) and (b) highlight of the short-term transient up to $2500 \mathrm{~s}$.

The dynamic response of the reactor following a reactivity insertion due to control rod withdrawal is now presented for the low-power $(50 \mathrm{~kW})$ and the high-power transients (200 $\mathrm{kW}$ ), in terms of the time evolution of the reactor power and of the main thermal-hydraulic quantities of interest. A comparison with the response of the previous model (Cammi et al., 2013) is carried out.

In general, the positive reactivity insertion due to control rod withdrawal causes a quick power increase, which leads to a system temperature increase. Consequently, the temperature feedback effects (Equation 18) cause an overall negative reactivity insertion. Whereas the original model of (Cammi et al., 2013) was able to predict the short-term behaviour of the reactor following such a transient, it fails at predicting the system response as the time of analysis increase, as seen in Figure 9. The reason of this discrepancy lies in the fact that the original model neglected all long-term effects that influence the reactor operation, such as the neutron poison accumulation due to absorption reactions within the fuel, and the water flow recirculation caused by the primary pool above the core.

With respect to the power and fuel temperature time evolution, the most striking difference lies in the fact that in the present model a new steady state is no longer reached following a reactivity insertion. As seen in Figure 1, during operation the overall concentration of neutron poisons species increases, thus causing an increase in terms of neutron absorption within the fuel. This causes a decrease in the number of fission events, and thus a decrease in power. Whereas in the original model the feedback effect of the fuel temperature coefficient caused a new steady state to be reached, in the long term the additional effect of neutron poisons accumulation causes the reactor to no longer reaches a stationary state following a reactivity insertion. Conversely, this power decrease is the cause for the slight increase in fuel temperature before decreasing. It is worth noting how the dynamic of the fuel temperature is much slower 
than both the power and the original model one, again likely due to the long-term effects of poison accumulation.

(a) Reactor Power

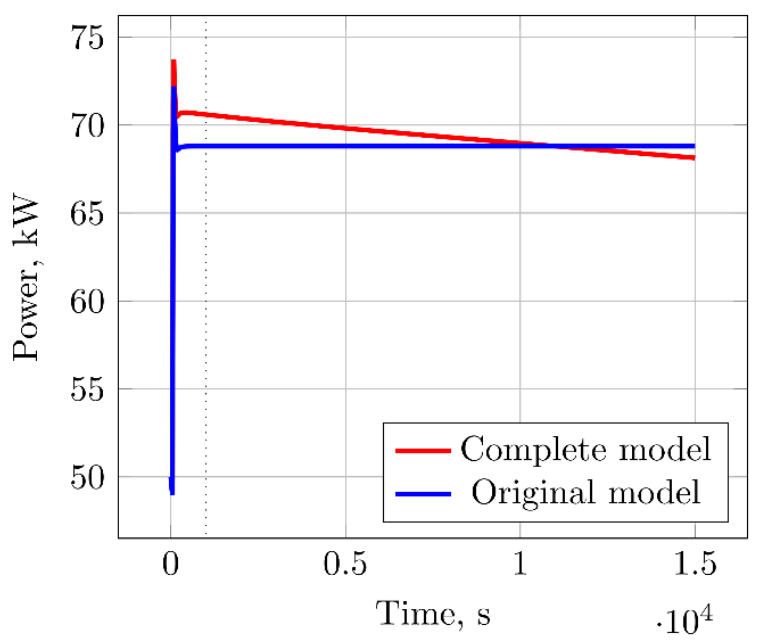

(c) Average Coolant Temperature

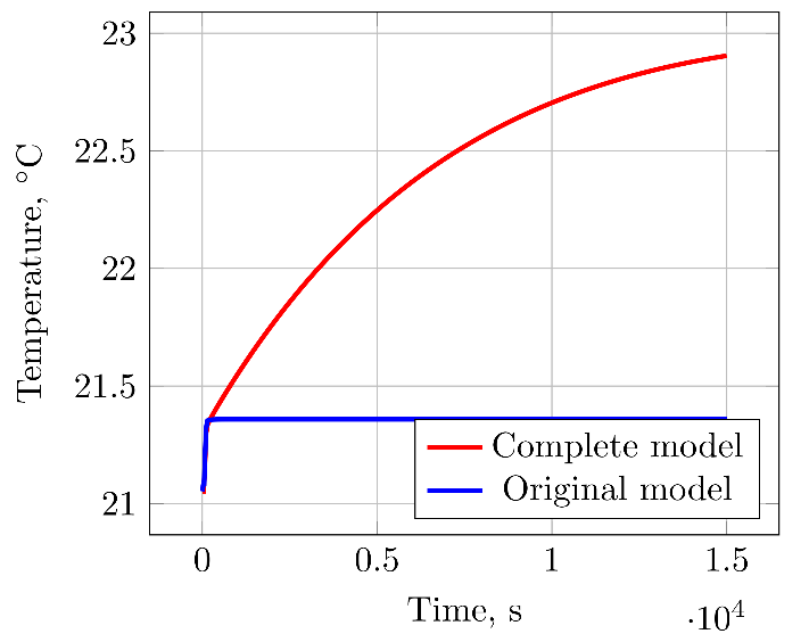

(b) Fuel Temperature

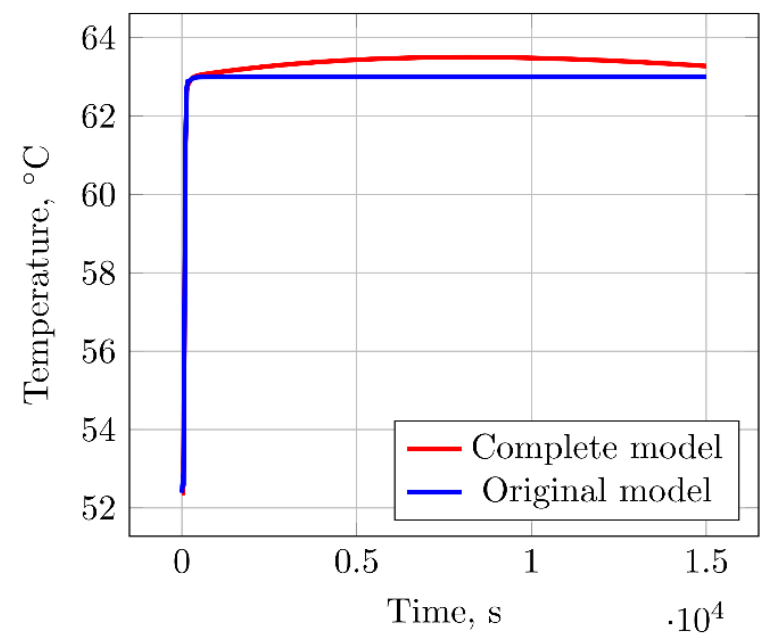

(d) Core Mass Flow Rate

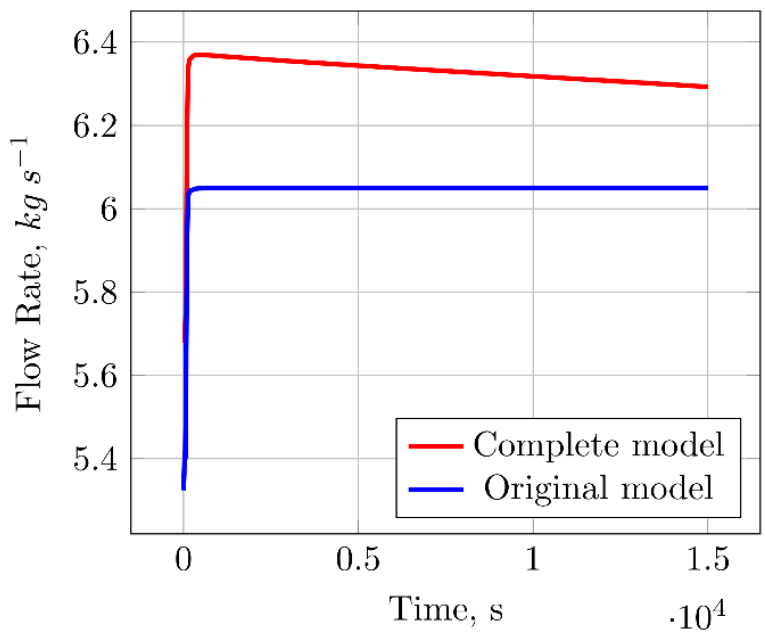

Figure 9: Long-term response of the reactor power (top left) and of the main thermo-hydraulics quantities (fuel temperature, average core coolant temperature, core mass flow rate) to reactivity insertion, at starting power of 50 $\mathrm{kW}$ (in red), and comparison with the original model (in blue).

The difference between the two models for what concerns the time evolution of the coolant temperature is much more significant than the one for the fuel temperature. In (Cammi et al., 2013), the temperature increase in the core was negligible, rising less than half degrees and quickly reaching a steady state. However, in the complete model not only this quantity does not reach a steady state, similarly to the fuel temperature, but its increase is much higher. Now, whereas in the older model the core coolant temperature was computed with respect to the inlet temperature, a fixed quantity, in the present work it is computed with respect to the average pool temperature, which is increasing as time goes on before eventually starting decrease. It is worth noting how the initial transient is much smaller than the one for the fuel, as well as how 
the dynamic of the coolant is slower. This slower dynamic can be explained both with the fact that the characteristic time constant $\tau$ for the coolant is lower than the one for the fuel. In addition, in the present work the temperature feedback coefficient for the coolant has been taken as positive, meaning that an increase in power causes an increase in coolant temperature. The presence of the primary pool also influences the behaviour of the core mass flow rate due to natural circulation. Not only the maximum value reached during the transient is higher, but again a stationary state is no longer obtained. Instead, the mass flow rate starts decreasing as the power decreases.

(a) Reactor Power

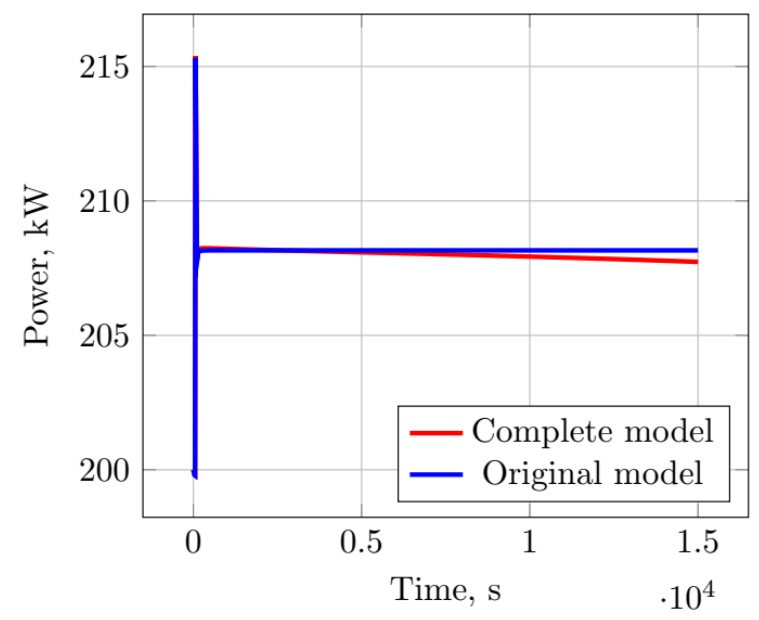

(c) Average Coolant Temperature

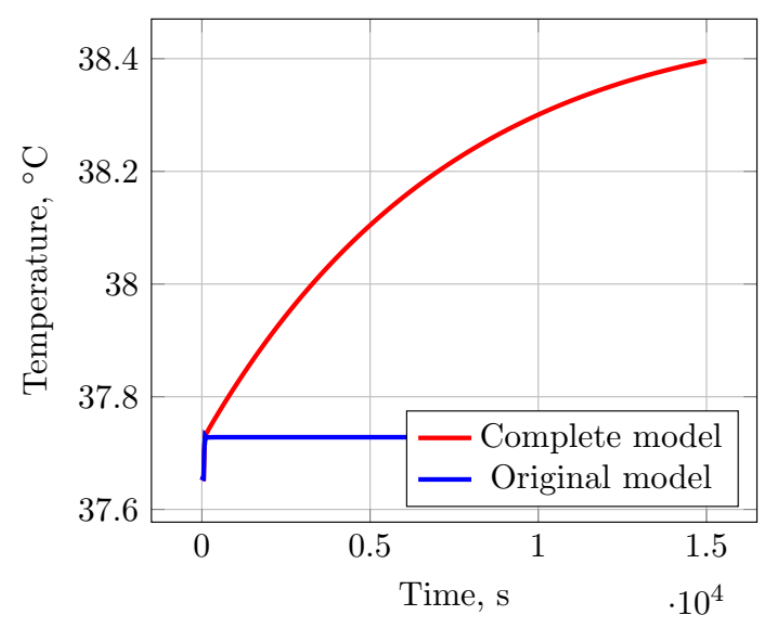

(b) Fuel Temperature

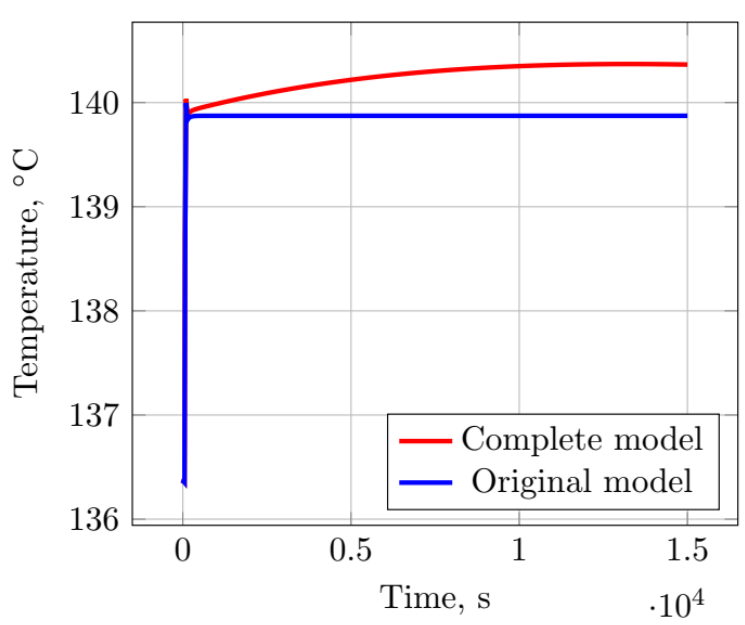

(d) Core Mass Flow Rate

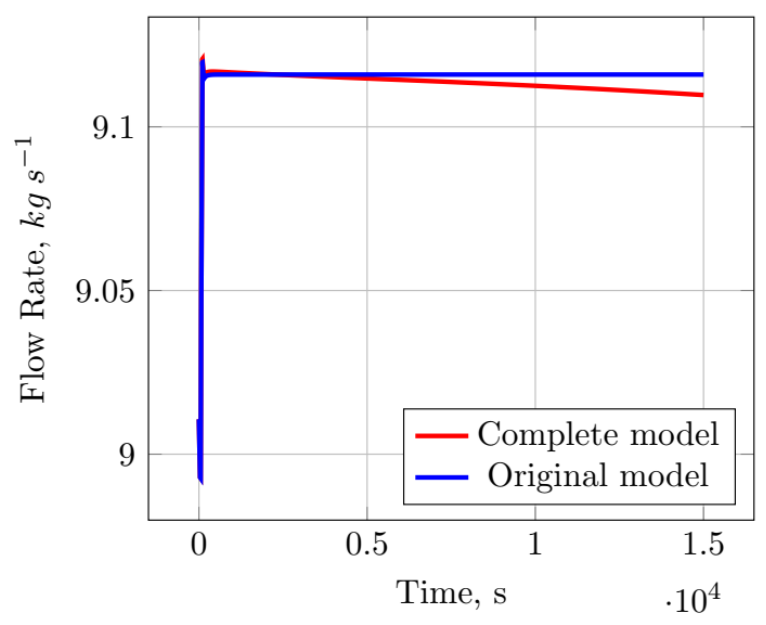

Figure 10: Long-term response of the reactor power (top left) and of the main thermo-hydraulics quantities (fuel temperature, average core coolant temperature, core mass flow rate) to reactivity insertion, at starting power of $200 \mathrm{~kW}$ (in red), and comparison with the original model (in blue).

Figure 10 reports the comparison between the two models with respect to a high-power transient. First, there are fewer differences between the two models for what concerns the reactor power and the mass flow rate. The latter can be explained by the fact that, as the coolant temperature increases, the water mass flow rate increases, and the Reynolds number gets higher, allowing the adopted heat transfer correlation to provide more accurate results. The former can 
be explained that, as the power increases, the importance of the fuel feedback coefficient remains higher than the neutron poisons feedback for a longer period of times. However, on the contrary, there are more discrepancies between the two models for what concerns the fuel and coolant temperature. The assumption of neglecting the coolant feedback coefficients, as did in (Cammi et al., 2013), is no longer accurate at higher power levels, where the coolant temperature difference is higher and thus their effect is more significant.

\section{Linear stability analysis}

It can be clearly seen how the system of equations presented in Section 2 is not linear. Indeed, the reactivity depends on the reactor power through the reactivity feedback coefficients. In addition, the core mass flow rate (equation 21), taken in this work as a state variable, depends both on the coolant and pool temperature, and the poison concentrations depend both on the fuel density and on the reactor power.

However, it is possible to draw some important conclusions about the nonlinear system by studying an associated linear approximation. As stated in (Ogata, 2002), the stability of a nonlinear system under small perturbations with respect to the stationary state can be studied. Therefore, it is necessary to express the variables of interest in terms of their variations with respect to the steady state (under the assumption of fresh fuel condition). Considering a general variable $q(t)$ :

$$
q(t)=q_{0}+\delta q(t)
$$

With respect to reactivity, the system is initially critical, and therefore $\rho_{0}=0$. The variation of reactivity due to the different feedback effects can be written as:

$$
\delta \rho(t)=\alpha_{\text {rod }} \delta h_{\text {rod }}+\alpha_{f} \delta T_{f}+\alpha_{c} \delta T_{c}+\alpha_{v} \delta \delta_{c}+\alpha_{X e} \delta X e+\alpha_{S m} \delta S m
$$

To linearize the model, a first order Taylor approximation (Marquez, 2003) is used:

$$
\begin{aligned}
f\left(x_{0}+\delta x, y_{0}\right. & +\delta y, \ldots) \\
& \approx f\left(x_{0}, y_{0}, \ldots\right)+\left(\frac{\partial f}{\partial x}\right)_{x_{0}, y_{0}, \ldots} \delta x+\left(\frac{\partial f}{\partial y}\right)_{x_{0}, y_{0}, \ldots} \delta y+\cdots
\end{aligned}
$$

Using this approximation, and neglecting the bilinear terms, the linearization of the equation system around the equilibrium state 0 is obtained: 


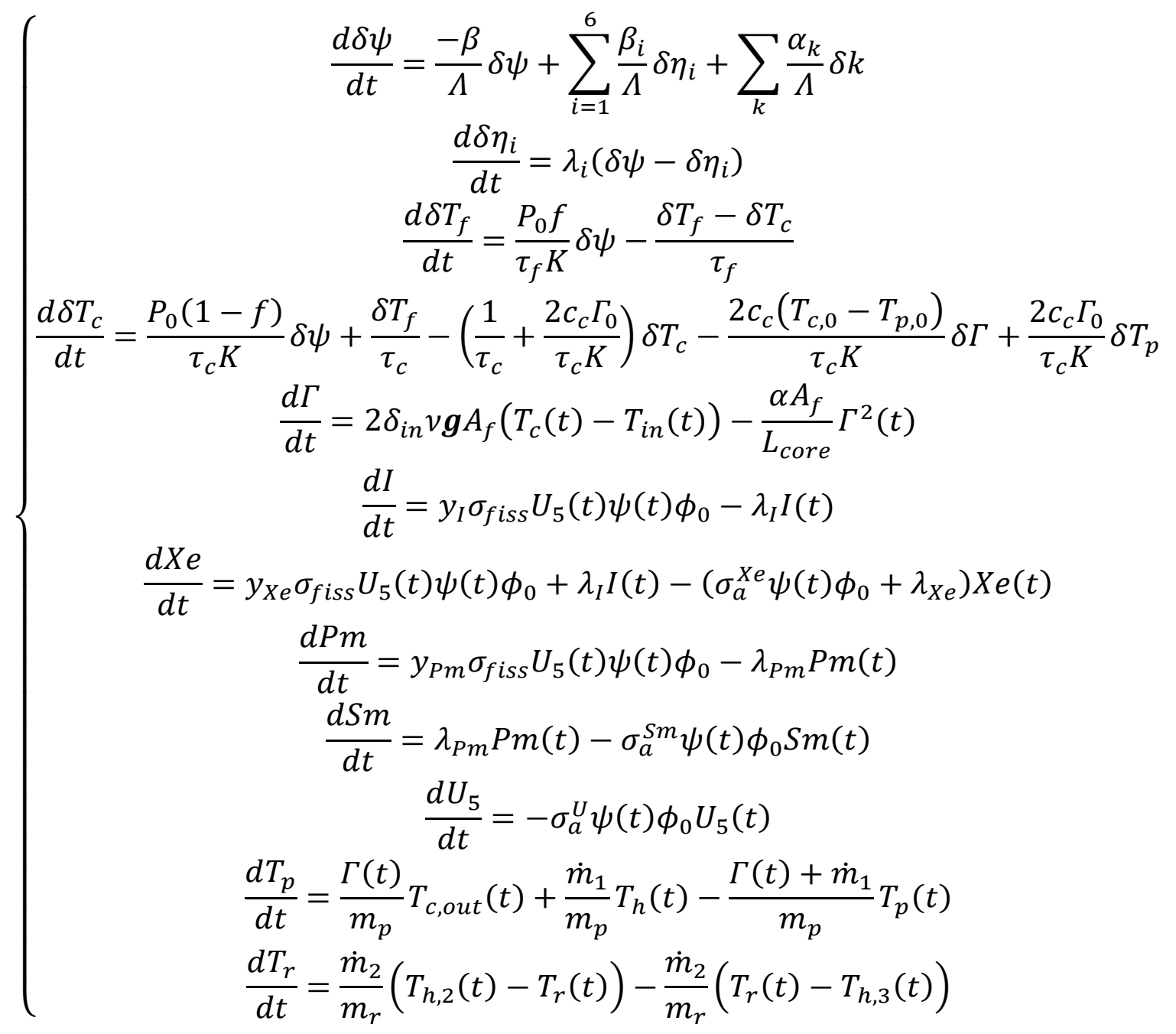

The above linear system can be written using the so-called state-space representation:

$$
\left\{\begin{array}{l}
\dot{\boldsymbol{x}}=f(\boldsymbol{x}, \boldsymbol{u})=\underline{\underline{\boldsymbol{A}}} \delta \boldsymbol{x}+\underline{\underline{\boldsymbol{B}}} \delta \boldsymbol{u} \\
\boldsymbol{y}=g(\boldsymbol{x}, \boldsymbol{u})=\underline{\underline{\boldsymbol{C}}} \delta \boldsymbol{x}+\underline{\underline{\boldsymbol{D}}} \delta \boldsymbol{u}
\end{array}\right.
$$

The matrix $\underline{\boldsymbol{A}}$ is the so-called state matrix or dynamic matrix, $\underline{\boldsymbol{B}}$ is the input or control matrix, $\underline{\underline{C}}$ is the output matrix, $\underline{\underline{D}}$ is the feed-forward matrix, and $\boldsymbol{y}$ is the output vector of the quantities of interest:

$$
\boldsymbol{y}=\left[\begin{array}{lllllll}
\psi & T_{f} & T_{c} & \Gamma & X e & S m & T_{p}
\end{array}\right]
$$

This formulation allows the study of the system stability from the eigenvalues of the dynamic matrix. For small variations, the heat transfer coefficient $K$, the feedback coefficients $\alpha_{k}$ and the fuel heat capacity $C_{f}$ are taken as independent from the state variables. Considering as input for the system the external reactivity inserted by extraction or insertion of the control rods, the open-loop transfer function of the linear system in state-space representation can be 
written as follows:

$$
G(s)=\frac{\delta \psi}{\rho_{\text {rods }}}=\underline{\underline{\boldsymbol{C}}}[s \underline{\underline{\boldsymbol{I}}}-\underline{\underline{\boldsymbol{A}}}]^{-1} \underline{\underline{\boldsymbol{B}}}
$$

The stability of the reactor has been analysed first using the root locus method for a fixed value of the feedback coefficients. In this method, the behaviour of the roots of the characteristic equation is investigated. A linear system is unstable when at least one root has positive real part.
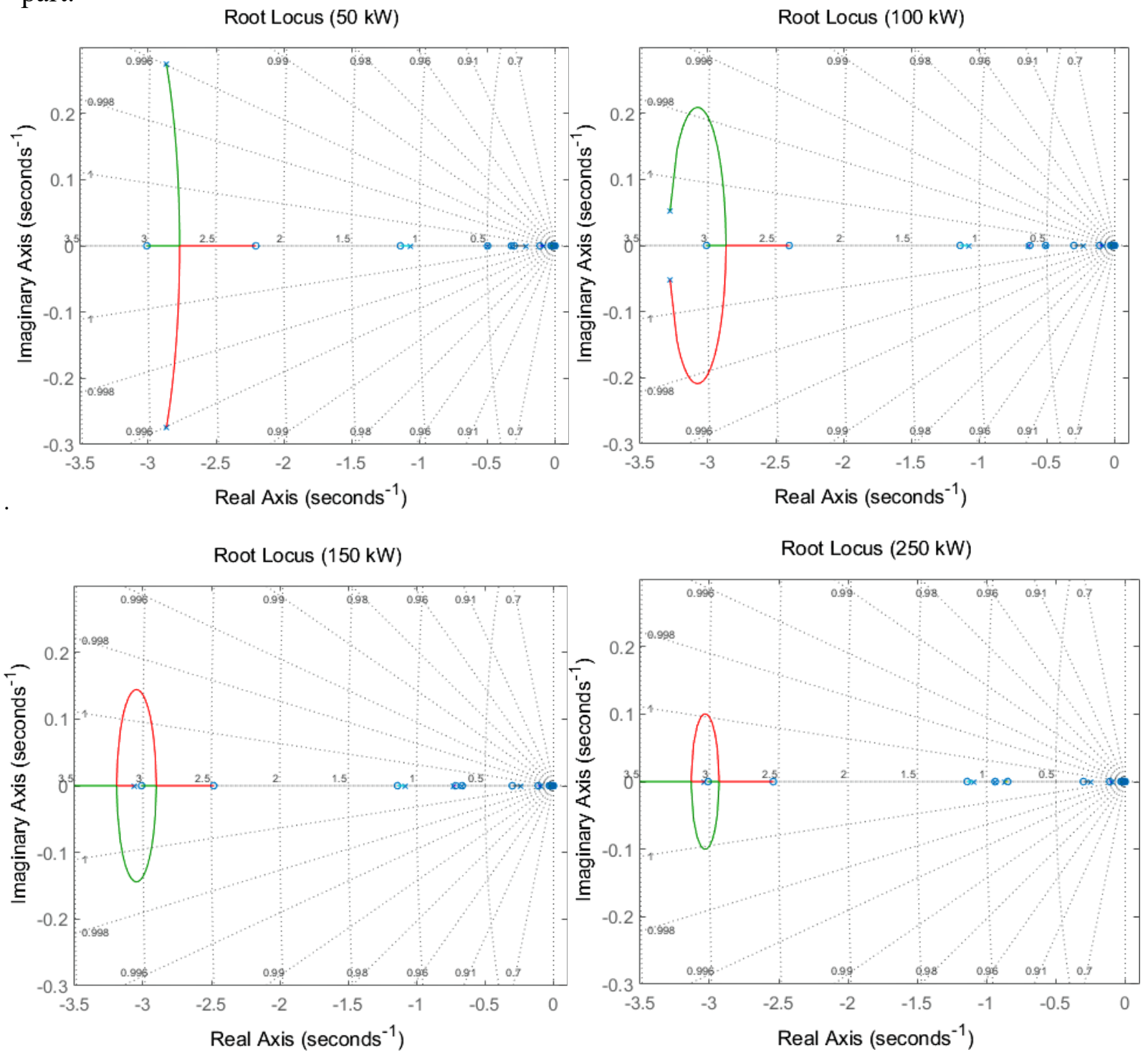

Figure 11: Poles of the system and detailed view of the root locus for the studied linear system at different power levels. Poles with imaginary part different than zero appears as the power level decreases

Figure 11 shows the root locus for the linear system at different power levels. It can be seen how poles with imaginary part different from zero appear as the power level decreases, contrary to what was obtained in (Cammi et al., 2013). The formation of fission products thus has a regulating effect on the dynamic of the system, reducing the frequency of oscillations as the 
power increases. Without the implementation of the neutron poison evolution in the model, the thermal feedback effects are such that the oscillation frequency drops as the power decreases, as seen in (Cammi et al., 2013). Overall, since all the poles have negative real part, the system remains stable at all power levels. Indeed, for this study, the two main feedback coefficients, namely the fuel temperature and the coolant temperature ones, are kept constant and equal to their nominal values. It is now interesting to study the reactor linear stability within the thermal feedbacks parametric space. To this end, the transfer function denominator (equation 51) is set to zero, with the thermal feedback coefficients $\alpha_{f}$ and $\alpha_{c}$ taken as variable parameters.

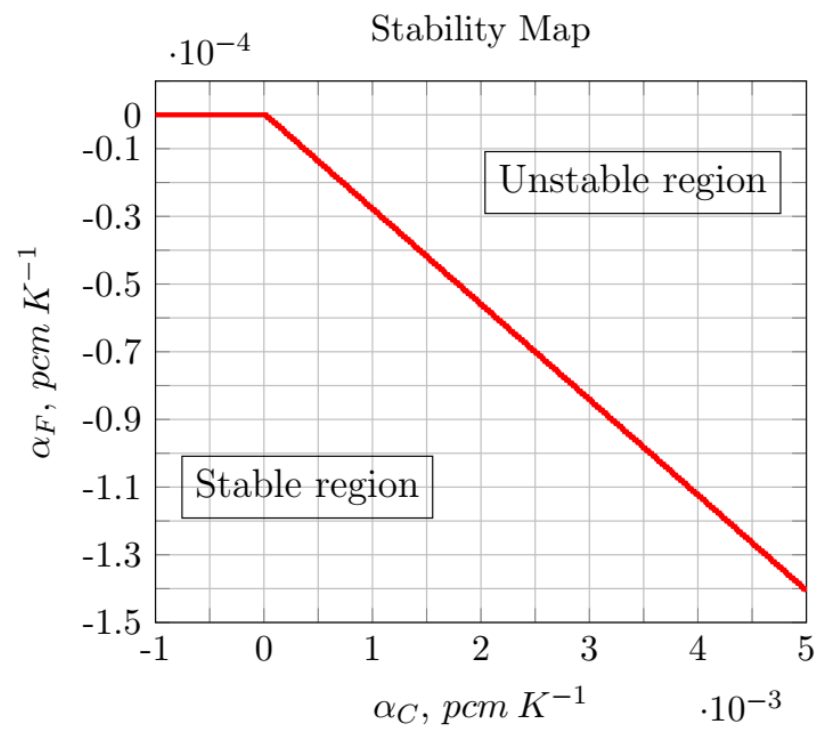

Figure 12: Stability map for the linear reactor model. Positive values for the coolant feedback coefficient are allowed, due to its less relevancy with respect to the fuel one.

Figure 12 shows the characterisation of reactor core stability within the thermal feedbacks parametric space. As expected, the reactor is unstable for positive values of $\alpha_{f}$. However, with respect to the coolant temperature feedback coefficients there exists some pairs of $\left(\alpha_{c}, \alpha_{f}\right)$, with the former positive and the latter negative, for which the reactor remains stable with respect to the linear analysis. This implies that the effect of the fuel temperature feedback coefficient prevails with respect to the coolant temperature coefficient, as already seen in Section 3, due to the higher increase in fuel temperature with respect to the coolant.

\section{Conclusions}

In this work, a zero-dimensional model for the TRIGA Mark II reactor of the University of Pavia was developed to simulate the complete dynamic behaviour. With respect to (Cammi et al., 2013), the mass flow rate evolution model, the neutron poisons evolution model and the treatment of the primary and secondary pool temperature were added, to simulate the long-term behaviour of the reactor following a reactivity transient. Validation was performed through a comparison with experimental data collected at the reactor facility. It is shown how the simulated responses at different power levels accurately reproduce the transient behaviour of the reactor, with observed discrepancies less than 5\%. These can be explained with the physical simplifications still present in the model, as well as with the fact that a 0D model has been used, 
instead of a full 3D one, especially for what concerns the neutronic aspects. The developed model is capable to reproduce the dynamic behaviour of the TRIGA reactor. In addition, a linear stability analysis was performed through the root locus method, highlighting the differences in dynamics at different power levels (namely, the oscillatory behaviour at low power levels due to the presence of poles with imaginary part different from zero). A stability map was also evaluated, studying the system stability for different values of the thermal feedback coefficients, whose value has not been unequivocally determined yet. The latter shows that the reactor remains stable even for positive values of the moderator feedback coefficient, confirming its low influence with respect to the fuel one. Despite the simplicity of a linear stability analysis such as the one performed in this work, the obtained results represent a step forward for the designing of a device for the reactor power control.

\section{Acknowledgements}

This research did not receive any specific grant from funding agencies in the public, commercial, or not-for-profit sectors.

Declaration of interests: none.

\section{References}

[1] P. D. Christofides, Nonlinear and robust control of PDE systems: Methods and applications to transport-reaction processes. In System \& Control: Foundations and Applications; Edition (1), Birkhauser, Basel, Switzerland, 2001.

[2] G. Pitton; G. Rozza, On the application of reduced basis methods to bifurcation problems in incompressible fluid dynamics. Journal of Scientific Computing, 2017, 73, 157-177.

[3] A. Cammi; R. Ponciroli; A. Borio di Tigliole; G. Magrotti; M. Prata; D. Chiesa; E. Previtali; A zero dimensional model for simulation of TRIGA Mark II dynamic response. Progress in Nuclear Energy, 2013, 68, 43-54.

[4] M. Johnson; S. Lucas; P. Tsvetkov, Modelling of reactor kinetics and dynamics. Technical report, Idaho National Laboratory, Idaho Falls (ID), USA (2010)

[5] M. H. Zahedi; G. R. Ansarifar, Estimation of the poisons reactivity in the PWR Nuclear Reactors using modified higher order sliding mode observer based on the multi-point nuclear reactor model. Annals of Nuclear Energy, 2018, 112, 158-169.

[6] T. Zagar; M. Ravnik, Positive temperature reactivity coefficient of a TRIGA reactor at room temperature, Kernetechnik, 2005, 70(4), 223-229.

[7] N. E. Todreas; M. S. Kazimi, Nuclear System I: Thermal-Hydraulic Fundamentals, Edition (3), Taylor \& Francis, Milton Park, Abdingdon-on-Thames, UK, 1990.

[8] F. W. Dittus; L. M. K. Boelter, Heat Transfer in Automobile Radiation of the Tubular Type. University of California Publications in Engineering 1930, 2, 443-461.

[9] D. Ruiz; A. Cammi; L. Luzzi, Dynamic stability of natural circulation loops for single-phase fluids with internal heat generation, Chemical Engineering Science, 2015, 126, 573-583.

[10] B. T. Swapnalee; K. Vijayan, A generalized flow equation for single phase natural circulation loops obeying multiple friction laws, International Journal of Heat and Mass Transfer, 2011, 54, 2618-2629.

[11] A. Pini, Analytical and numerical investigation of single-phase natural circulation dynamics in presence of distributed heat sources, Ph.D. thesis, Politecnico di Milano, Milano, Italy (2017).

[12] S. Boarin; A. Cammi; R. Ponciroli; D. Chiesa; E. Previtali; M. Sisti; G. Magrotti; M. Prata; A. Salvini, 
Object-Oriented Modelling and simulation of a TRIGA reactor plant with Dymola, Energy Procedia, 2016, 101, 42-49.

[13] The MathWorks, Inc., SIMULINK Dynamic System Simulation Language User Guide (2008).

[14] L. F. Shampine, Numerical Solution of Ordinary Differential Equations, Chapman \& Hall, London, UK, 1994.

[15] A. Borio di Tigliole; A. Cammi; A. Chiesa; M. Sisti, TRIGA reactor absolute neutron flux measurement using activated isotopes. Progress in Nuclear Energy, 2014, 70, 249-255.

[16] K. Ogata, Modern Control Engineering, Prentice Hall, Upper Saddle River (NJ), USA, 2002.

[17] H. J. Marquez, Nonlinear control systems: analysis and design, John Wiley \& Sons, Hoboken (NJ), USA, 2003. 